\title{
EVOLUTION OF THE COLOUR-MAGNITUDE RELATION OF ELLIPTICAL GALAXIES
}

\author{
TADAYUKI KODAMA ${ }^{1,2}$, NOBUO ARIMOTO $^{2}$, AMY BARGER $^{1,3}$ \\ AND \\ ALFONSO ARAGÓN-SALAMANCA ${ }^{1}$ \\ ${ }^{1}$ Institute of Astronomy, University of Cambridge, Madingley \\ Road, Cambridge, CB3 OHA, UK \\ 2 Institute of Astronomy, University of Tokyo, Mitaka, Tokyo \\ 181, Japan \\ ${ }^{3}$ Institute for Astronomy, University of Hawaii, 2680 Woodlawn \\ Drive, Honolulu, Hawaii 96822, USA \\ e-mail: kodama@ast.cam.ac.uk
}

This paper presents the quantitative analyses of evolution of the colourmagnitude (C-M) relation of early type galaxies in 9 distant clusters (AC $103[\mathrm{z}=0.31$ ], AC 114 [0.31], AC 118 [0.31], Cl 0024+16 [0.39], Cl 041265 [0.51], Cl 0016+16 [0.546], Cl 0054-27 [0.563], GHO 1603+43 [0.895], and 3C 324 [1.206]) at $0.3<z<1.2$ based on the evolutionary model of elliptical galaxies (Kodama \& Arimoto 1997, A\&A, 320, 41). The model is calibrated to reproduce the C-M relaiton of Coma ellipticals at $z=0$ (Bower, Lucey, Ellis 1992, MNRAS, 254, 589) and gives the evolution of slope and zero-point of the C-M relation as a function of redshift. The slopes of $\mathrm{C}-\mathrm{M}$ relation can be reproduced by a single model sequence in which mean stellar metallicities of ellipticals are varied as a function of galaxy luminosity and ages are assumed to be equally old. The zero-points of C-M relation of the two most distant clusters (GHO 1603+43, 3C 324) put constraint on the epoch of major star formation in early type galaxies that should be $z>2-4$. The cluster early type galaxies are suggested to be uniformly old and to have universal metallicity sequence as a function of galaxy mass. We find no significant difference of $\mathrm{C}-\mathrm{M}$ relation among the different clusters. This will put strong constraint on the formation of clusters of galaxies as well, since they must have shared a common formation mechanism to produce the homogeneous C-M relation. 